\title{
Environmental Tobacco Smoke Exposure and Brain Functioning Associated with Smoking Cue-Reactivity and Inhibitory Control in Nonsmoking Adolescents
}

\author{
Anna Johanna Maria Gemma Boormans ${ }^{a, b}$ Joyce Dieleman ${ }^{b, c}$ \\ Marloes Kleinjan ${ }^{c, d}$ Roy Otten ${ }^{b}$ Maartje Luijten ${ }^{b}$

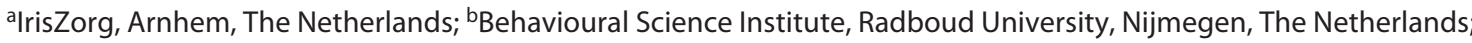 \\ 'Department of Child and Adolescent Mental Health, Trimbos Institute, Utrecht, The Netherlands; Interdisciplinary \\ Social Science, Utrecht University, Utrecht, The Netherlands
}

\section{Keywords}

Environmental tobacco smoke exposure · Smoking

cue-reactivity $\cdot$ Inhibitory control $\cdot$ Adolescence .

Neuroimaging

\begin{abstract}
Introduction: Despite its well-established negative effects, environmental tobacco smoke (ETS) exposure remains highly prevalent worldwide. ETS exposure is associated with a wide range of physical and mental health-related problems among youth, including an increased likelihood to develop nicotine dependence. Up till now, neurocognitive effects of ETS exposure are largely unknown, while such effects could explain the role of ETS exposure in the development of nicotine dependence. Therefore, this preregistered study investigated the role of current ETS exposure in brain functioning associated with smoking cue-reactivity and inhibitory control. Method: Concurrent with functional magnetic resonance imaging, nonsmoking adolescents aged $14-18$ years $(N=51)$ performed a smoking cue-reactivity task, assessing brain functioning to smoking cues, and a Go/NoGo task measuring inhibitory control. ETS exposure was measured using a self-report questionnaire and biochemically verified. $\boldsymbol{R e}$ -
\end{abstract}

karger@karger.com www.karger.com/ear

Karger $\stackrel{\text { ' }}{5}$

马OPEN ACCESS
(C) 2021 The Author(s)

Published by S. Karger AG, Basel

This is an Open Access article licensed under the Creative Commons Attribution-NonCommercial-4.0 International License (CC BY-NC) (http://www.karger.com/Services/OpenAccessLicense), applicable to the online version of the article only. Usage and distribution for commercial purposes requires written permission. sults: No significant associations were observed between current ETS exposure and brain functioning associated with smoking cue-reactivity and inhibitory control. Conclusion: These findings suggest that low-to-moderate levels of current ETS exposure are not associated with increased salience of smoking cues or deficits in inhibitory control in nonsmoking adolescents. Longitudinal research is needed to further clarify the exact effect of lifetime ETS exposure on brain functioning, as well as research focusing on the effects of higher levels of ETS exposure.

(c) 2021 The Author(s)

Published by S. Karger AG, Basel

\section{Introduction}

Despite the existent knowledge about the toxic contents of tobacco smoke, children and adolescents are still frequently exposed to environmental tobacco smoke (ETS) worldwide [1]. In 2015, 21\% of Dutch adolescents between 13-18 years old were exposed to ETS in their homes [2]. ETS exposure is associated with a range of adverse health and behavioral effects [3-5]. Young people

A.J.M.G. Boormans and J. Dieleman have contributed equally. 
are particularly vulnerable to the effects of ETS, as they display higher cotinine and nicotine levels during and after exposure of ETS than adults, indicating that youth might absorb more nicotine [6, 7]. In addition, ongoing brain maturation [8] may render adolescents vulnerable to effects of ETS exposure on brain functioning $[9,10]$. Studies have shown that prenatal cigarette exposure causes neurocognitive changes in youth $[11,12]$. However, it is unknown whether ETS exposure during adolescence results in similar functional brain changes. Therefore, this study investigated the relation between ETS exposure and brain functioning among nonsmoking adolescents using functional magnetic resonance imaging (fMRI).

In addition to the poor health outcomes associated with ETS exposure, nicotine uptake caused by ETS exposure might prompt increased sensitivity to nicotine, which in turn may increase the likelihood for smoking initiation, continuation, and nicotine dependence [13, 14]. Specifically, one study in 9-12-year-old nonsmokers showed that $6 \%$ reported experience of craving for cigarettes and $8 \%$ reported cue-triggered wanting to smoke in response to ETS [15]. Moreover, there is evidence that ETS could cause plasma nicotine concentrations equivalent to levels produced by tobacco smoking $[16,17]$. To test whether nicotine inhaled from ETS exposure crosses the blood-brain barrier, Brody et al. [18] used positron emission tomography scanning after ETS exposure and showed that nicotinic acetylcholine receptors were occupied as a result of ETS exposure in adult smokers and nonsmokers. Together this growing body of evidence points to increased susceptibility to smoking caused by ETS exposure, although underlying mechanisms are unknown. Understanding these mechanisms could provide information on how ETS exposure renders adolescents vulnerable for smoking initiation and dependence.

While the effects of ETS exposure on neurocognitive functioning are unknown, neurocognitive mechanisms related to active smoking have been examined extensively. Studies indicated differential brain activation patterns between smokers and nonsmokers when exposed to smoking pictures (i.e., smoking cue-reactivity) [19-21] and when implementing inhibitory control $[22,23]$. Both cue-reactivity and inhibitory control are incorporated in addiction models and are relevant for the development and continuation of addiction [24,25]. Overall, addiction is characterized by an overactive motivational and reward network during confrontation with substance-related cues $[21,26]$. After repeated drug use, substance-related cues become conditioned cues and activate the mesolimbic dopamine system [27]. A cue-reactivity meta-analysis
[20] indicated that smoking cues evoke larger neural responses than neutral cues within anterior cingulate cortex (ACC), dorsal prefrontal cortex, medial prefrontal cortex, insula, dorsal striatum, the visual system, precuneus, and posterior cingulate gyrus. Another meta-analysis found reactivity in the bilateral ventral striatum, ACC, left amygdala, and left temporoparietal junction in response to smoking cues [21]. These studies indicate that nicotine dependence is associated with increased activity in the motivational network.

Besides the overactive motivational network, addiction is characterized by impaired cognitive control [24, 28]. Previous research demonstrated that smoking is associated with behavioral deficits in inhibitory control [23, $29,30]$. In line with these behavioral impairments, reduced inhibitory control is also reflected in decreased brain activation in the inhibitory control network. Specifically, reduced activation in the dorsal ACC [30, 31], the right superior frontal gyrus, left middle frontal gyrus, bilateral inferior parietal lobule, and middle temporal gyrus has been found in smokers compared to nonsmokers [30]. Moreover, lower activation during inhibitory control in the supplementary motor area (SMA) was associated with higher levels of nicotine dependence [32]. Finally, the involvement of putamen-insula interactions was highlighted in smokers relative to nonsmokers during inhibitory control [33]. Effects of prenatal tobacco exposure on inhibitory control have also been observed showing altered neurocognitive functioning in areas related to response inhibition $[11,12,34]$. Overall, nicotine dependence is linked to behavioral deficits and reduced brain activity in brain regions implicated in the inhibitory control network.

Up till now, it is unclear whether neurocognitive deficits related to smoking cue-reactivity and inhibitory control in smokers could also be observed in response to ETS exposure. Hence, the goal of this study was to examine the association between current ETS exposure and brain functioning associated with smoking cue-reactivity and inhibitory control in nonsmoking adolescents using fMRI. We hypothesized that higher levels of ETS exposure would be associated with increased brain activity toward smoking-related cues in the striatum, insula, amygdala, ventromedial prefrontal cortex (VMPFC), and ACC $[20,21]$. We further expected that ETS exposure would be associated with reduced inhibitory control, meaning more difficulty to inhibit their response, and a decrease of brain activity in brain regions implicated in inhibitory control, that is, the putamen, SMA, IFG, ACC, and DLPFC $[22,33,34]$. 


\section{Methods}

\section{Participants}

Fifty-one Dutch nonsmoking adolescents aged 14-18 years were included. Two participants were excluded, one due to incomplete task administration and one due to an outlying value on ETS exposure, resulting in a total of 49 participants $\left(\mathrm{M}_{\mathrm{age}}=16.47\right.$, $\mathrm{SD}=1.16,30.6 \%$ men). Participants were recruited through social media, secondary schools, and vocational education. Exclusion criteria were (a) ever smoked a complete cigarette or more, (b) use of psychoactive medication that cannot be stopped for $24 \mathrm{~h}$, (c) fMRI contraindications, and (d) history of neurological disease. Informed consent was obtained from all participants and from parents for participants younger than 16 years old. The study was approved by the ethical committee CMO Arnhem-Nijmegen (project \#2016-2409, 2015-2120). For more details on our study procedures, materials, and analyses, see our preregistration (https://osf.io/urhnv/).

\section{Materials}

\section{ETS Exposure}

ETS exposure was assessed using the following 2 questions: "Does your XXXX (e.g., [step]mother) smoke when she is around you?", response options were "yes" and "no". If the answer was yes, the following ETS exposure question was assessed: "How often does your XXXX smoke when you are around?", response options ranged from (0) my XXXX smokes, but not when I am around to (8) more than 5 times a day. Scores of 6 potential sources of ETS exposure (mother, father, siblings, best friend, friends, and others) were summed to determine the total amount of current ETS exposure. Higher scores on this questionnaire indicate more exposure to ETS. Self-report measures of ETS exposure are found to be a reliable measure in children as children are reliable reporters of the smoking behavior in their environment [35].

Saliva samples were collected to measure levels of cotinine, a metabolite of nicotine, to biochemically establish ETS exposure. Cotinine was analyzed using liquid chromatography coupled with mass spectrometry, with a quantification limit of $<1.0 \mu \mathrm{g} / \mathrm{L}$. Kendall's tau correlation was used to test whether cotinine in saliva was associated with the self-reported ETS exposure. Indeed, cotinine was significantly associated with the ETS exposure measure $\left(r_{\mathrm{T}}=\right.$ $0.259, p=0.027$ ). The small effect size of this correlation may be explained by the fact that cotinine levels in saliva only capture ETS exposure in the last $72 \mathrm{~h}$ and due to the lack of smoking by participants themselves [36].

\section{Pubertal Status}

To account for individual differences in ongoing hormonal development, participants filled out the Pubertal Development Scale (PDS) [37].

\section{Familial Risk}

Familial risk was included to account for familial vulnerability for nicotine dependence [38]. A composite measure was used in which the parent that accompanied the participant to the research session filled out a series of questions. First, the Fagerström Test for Nicotine Dependence (FTND) [39] was filled out for both parents representing the period in which they smoked heaviest, now or in the past $[39,40]$. Also, current smoking behavior of parents was rated in 4 categories (0) nonsmoking, (1) smoking $<10$ cigarettes per day, (2) smoking 10-19 cigarettes per day, and (3) smoking 20 or more cigarettes per day. Finally, smoking behavior of their own parents (i.e., grandparents of the participants) was rated. This category was rated 0 if they were nonsmokers, ex-smokers, or smoked $<10$ cigarettes a day and scored 1 if they currently smoke $>10$ cigarettes a day. For both parents, scores from the 3 domains were summed, resulting in 2 total scores (father and mother), which were subsequently summed and averaged, resulting in 1 score representing familial risk on a scale from 0 to 16 .

Alcohol Use

Problematic alcohol use was measured with the Alcohol Use Disorders Identification Test (AUDIT) [41].

Cognitive Tasks

The smoking cue-reactivity task (A) and the inhibitory control task (Go/NoGo) (B) are displayed and explained in Figure 1. Participants were not exposed to ETS for at least 3 and $2.5 \mathrm{~h}$ before task performance, respectively.

\section{fMRI Data Acquisition}

fMRI data were acquired on a Siemens 3 Tesla Skyra MRI scanner (Siemens Medical Systems, Erlangen, Germany) using a 32-channel coil. Functional T2*-weighted imaging was obtained using multiecho echo-planar imaging to acquire 39 axial slices in interleaved ascending order (voxel size, $3.5 \times 3.5 \times 3.0 \mathrm{~mm}$; matrix, $64 \times 64$; repetition time (TR), 2,020 ms; echo times (TE), 7, 16.3, 26,35 , and $44 \mathrm{~ms}$; flip angle, $80^{\circ}$ ). A high-resolution weighted anatomical scan was also obtained (MPRAGE; 192 axial slices; voxel size, $1 \times 1 \times 1 \mathrm{~mm}$; matrix, $256 \times 256$; TR, 2,300 ms; TE, $3.03 \mathrm{~ms}$; flip angle, $8^{\circ}$ ).

\section{Data Analysis}

\section{Behavioral Analyses}

Behavioral analyses were performed using the Statistical Package for the Social Sciences (SPSS, version 23). Outliers (3 SD) for each variable from questionnaire and behavioral data were removed, and missing questionnaire data were imputed with the mean. Kendall's tau correlations were computed between ETS exposure, pubertal status, familial risk, and NoGo accuracy to assess binary relationships. Subsequently, all predictors were mean-centered and a hierarchical linear regression analysis was performed to examine the effect of ETS exposure on NoGo accuracy with pubertal status and familial risk as control variables.

\section{fMRI Data Analyses}

Preprocessing steps were conducted in SPM12 (www.fil.ion. ucl.ac.uk/spm). Preprocessing of the functional data included realignment, echo combination using the PAID method [42], coregistration, and normalization into MNI space and smoothing with a FWHM of $8 \mathrm{~mm}$. After preprocessing, an independent component analysis-based automatic removal of motion artifacts was applied using FSL (www.fmrib.ox.ac.uk/fsl; ICA-AROMA) [43, 44].

Subsequently, data were modeled using a general linear model. The smoking cue-reactivity task was modeled with separate regressors for smoke, neutral, and romantic conditions. The Go/NoGo task was modeled with regressors for NoGo, Go, and IfGo trials separately for correct and incorrect responses. For both tasks, a nuisance regressor for instructions was modeled. The various 


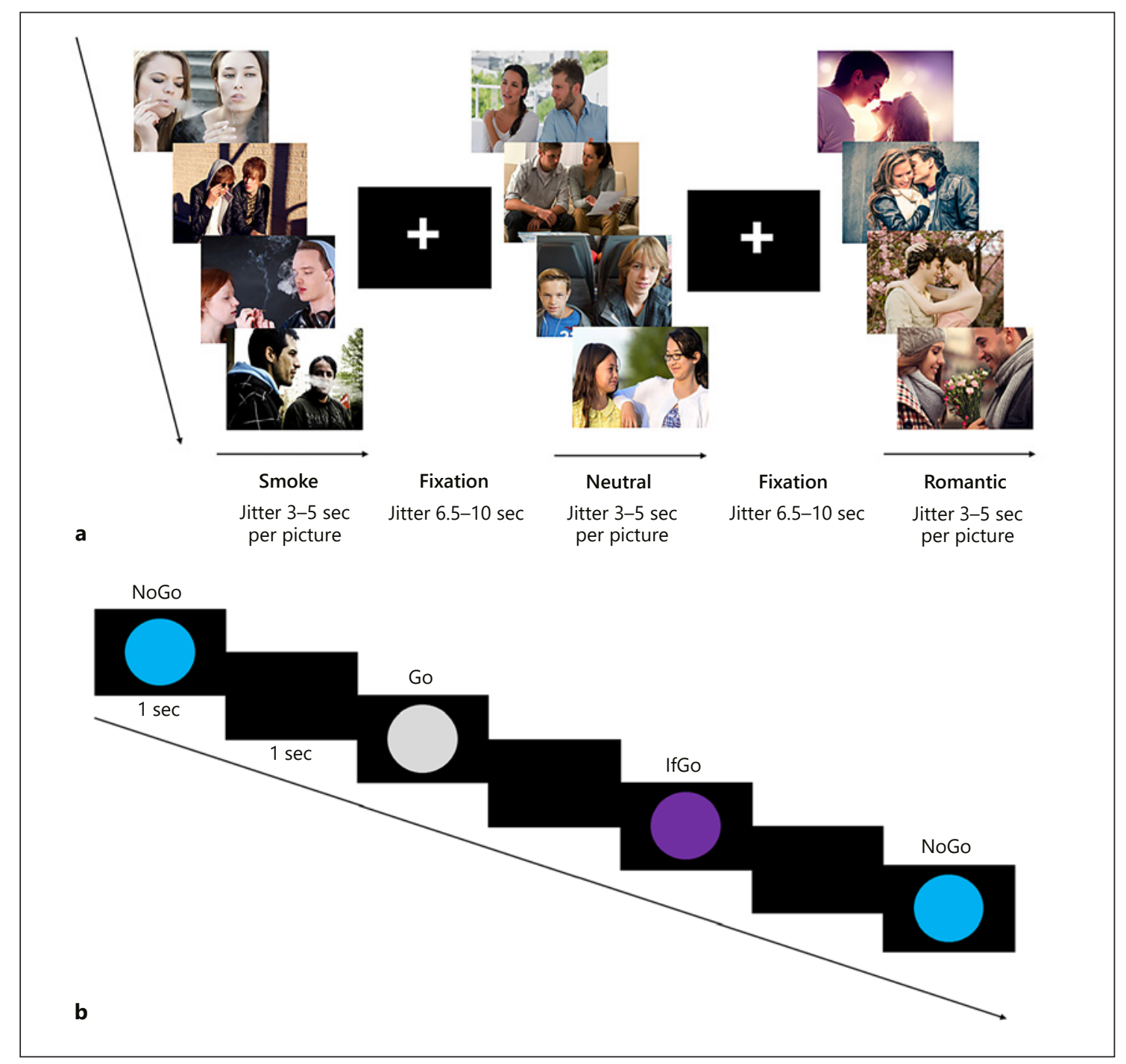

Fig. 1. Cognitive tasks. a Smoking cue-reactivity task: participants were instructed to pay attention to the content of the pictures and press a button when a new picture appeared on the screen. Thirtytwo photographs showing smoking-related pictures, 32 nonsmoking-related pictures, and 32 romantic pictures were presented. Images were presented in a block design. The task consisted of 24 blocks, with each block including 4 pictures from the same catego- ry. b Go/NoGo task: participants were instructed to respond to the Go and IfGo trials by pressing a button with the right thumb as fast as possible. They had to withhold their response, by not pressing the button, in NoGo trials. The task consisted of 383 trials, 249 $(65 \%)$ of the trials were Go trials, 67 (17.5\%) were IfGo trials, and 67 (17.5\%) were NoGo trials. blocks of the smoking cue-reactivity were modeled as boxcars with a duration of $16 \mathrm{~s}$ time-locked to the onset of the first picture in each block. The stimuli of the Go/NoGo task were modeled with a boxcar function (duration of 1,000 ms) time-locked to the onset of the stimuli. A temporal high-pass filter of $128 \mathrm{~s}$ was applied to remove low-frequency noise.

As preregistered, we first checked whether the tasks activated the expected brain networks. Therefore, second-level analyses including a whole brain one-sample $t$ test combining the smoke and neutral pictures as well as a whole brain one-sample $t$ test for the No_Gocorrect > Go_correct contrast were performed across all participants. Whole brain T-maps based on the one-sample $t$ tests were thresholded with a voxel-level uncorrected $p<0.001$, combined with a cluster-level family-wise error (FWE) corrected $p<$ 0.05 , accounting for multiple comparisons across the whole brain $[45,46]$.

Second, to investigate the effect of ETS exposure, brain multiple linear regressions on the smoke $>$ neutral and NoGocorrect $>$ Gocorrect contrasts were performed with pubertal status and familial risk as additional covariates. All predictors were first meancentered. These whole brain maps were thresholded with a voxellevel uncorrected $p<0.005$, combined with a cluster-level FWE 
Table 1. Descriptive statistics

\begin{tabular}{lrlc}
\hline Variable & Mean & SD & Range \\
\hline $\begin{array}{l}\text { Demographics } \\
\text { Sex }\end{array}$ & & & \\
$\quad$ Male, \% & 30.6 & - & - \\
Age & 16.47 & 1.16 & $14-18$ \\
Educational level, ${ }^{\text {a } \%}$ & & & \\
$\quad$ Low & 51 & - & - \\
$\quad$ Middle & 22.4 & - & - \\
$\quad$ High & 26.5 & - & - \\
ETS exposure** & 7.51 & 6.80 & $0-30$ \\
Saliva cotinine & 0.37 & 0.59 & $0-2.60$ \\
Pubertal status & 3.54 & 0.39 & $2.6-4$ \\
Familial risk & 2.99 & 2.81 & $0-10.5$ \\
AUDIT & 5.02 & 5.02 & $0-22$ \\
\hline
\end{tabular}

$n=49$. AUDIT, Alcohol Use Disorder Identification Test. ** $n=48 .{ }^{\text {a }}$ Educational level: low includes MBO (senior secondary vocational education)/VMBO (preparatory secondary vocational education), middle includes HAVO (school of higher general secondary education), and high includes VWO (preuniversity education). ${ }^{\mathrm{b}} \mu \mathrm{g} / \mathrm{L}$.

corrected $p<0.05$, accounting for multiple comparisons across the whole brain $[45,46]$.

More exploratory, we also performed a whole brain one-sample $t$ test on the smoke $>$ neutral contrast to test whether there is an effect of smoking-related pictures independent of ETS exposure among nonsmoking adolescents. The whole brain one-sample sample $t$ test $T$-map was thresholded with a voxel-level uncorrected $p<0.001$, combined with a cluster-level FWE corrected $p<0.05$. Three participants were excluded from the imaging analyses for the smoking cue-reactivity task, and 4 participants were excluded for the Go/NoGo task because of missing/incomplete data or acquisition problems.

To further test the effect of ETS exposure on brain activation, small volume corrections (SVCs) were performed in predefined regions of interest (ROI). In order to do so, the results were first thresholded with a whole brain voxel-level of $p<0.005$ uncorrected and then corrected using a small volume cluster-level correction ( $p<0.05$ FWE corrected) in which the search volume was defined by the ROI. ROIs for smoking cue-reactivity included the bilateral striatum, ACC, VMPFC, insula, and amygdala. ROIs for inhibitory control included ACC/pre-SMA, DLPFC, IFG, and putamen. Except for the ventral part of the striatum for smoking cue-reactivity, all ROIs are defined based on the AAL-atlas [47].

Finally, we performed Bayesian statistics with default priors in JASP [48] to quantify the evidence supporting the null hypothesis in the case of nonsignificant frequentist statistics for the SVCs within ROIs. Applying Bayesian analyses on small volumes is not yet implemented in SPM, and therefore, we extracted the beta weights from the ROIs and exported these values to JASP to conduct the Bayesian analyses. Bayesian statistics are displayed by means of Bayes factors. The Bayes factor referred to as BF01 expresses relative evidence for the null hypothesis $(\mathrm{H} 0)$ compared to

Environmental Tobacco Smoke Exposure
Table 2. Correlation matrix

\begin{tabular}{lccll}
\hline & 1 & 2 & 3 & 4 \\
\hline 1. ETS exposure & - & 0.43 & 0.02 & 0.34 \\
2. Pubertal status & -0.08 & - & 0.22 & 0.07 \\
3. Familial risk & $0.24^{*}$ & -0.13 & - & 0.18 \\
4. NoGo accuracy & -0.10 & 0.19 & -0.14 & - \\
\hline
\end{tabular}

ETS, environmental tobacco smoke. ${ }^{*} p<05$; correlation coefficients (below diagonal) and their $p$ values (above diagonal).

the alternative hypothesis (H1). Even though the Bayes factor has a continuous scale, it is useful to summarize the Bayes factor in terms of discrete categories of evidential strength, see Wagenmakers et al. [49] for the classification scheme.

\section{Results}

\section{Descriptive Statistics}

Table 1 shows participants' characteristics and questionnaire data. Kendall's tau correlations showed that ETS exposure was positively and significantly related to familial risk $\left(r_{\mathrm{T}}=0.240, p=0.022\right.$; Table 2$)$. All other variables were not significantly related to one another. Table 3 shows accuracy and reaction times for both the cuereactivity task as well as the inhibitory control task.

\section{Behavioral Results}

The hierarchical linear regression analysis showed a nonsignificant regression equation in the first step ( $p=$ 0.151; Table 4). ETS exposure did not explain additional variance for NoGo accuracy $\left(\Delta R^{2}=0.025, p=0.273\right)$. This suggests that there is no association between ETS exposure and NoGo accuracy after controlling for pubertal status and familial risk. For the same analysis, $\mathrm{BF}_{01}=1.42$ was observed, indicating anecdotal evidence for the null hypothesis.

\section{Imaging Results}

All T-maps of the results discussed can be accessed at https://neurovault.org/collections/8086/.

\section{Smoking Cue-Reactivity}

First, we examined brain responses for the combination of smoking and neutral pictures to evaluate whether our task activated the expected brain regions. The results show that the expected brain network, consisting of both visual and mesolimbic and frontal regions, was activated 
Table 3. Accuracy and reaction times for smoking cue-reactivity task and Go/NoGo task

\begin{tabular}{|c|c|c|c|c|}
\hline \multirow[t]{2}{*}{ Cognitive task } & \multicolumn{2}{|l|}{ Accuracy } & \multicolumn{2}{|l|}{ Reaction time } \\
\hline & $\mathrm{M}(\mathrm{SD})$ & range & $\mathrm{M}(\mathrm{SD})$ & range \\
\hline \multicolumn{5}{|c|}{ Smoking cue-reactivity ${ }^{\mathrm{a}}$} \\
\hline Neutral & $99.32 \%(2.81)$ & $84.38-100$ & $564.36(210.98)$ & $308-1,204$ \\
\hline Smoke & $98.91 \%(3.11)$ & $84.38-100$ & $568.53(192.70)$ & $323-1,010$ \\
\hline Romantic & $99.25 \%(2.54)$ & $84.38-100$ & $551.55(208.28)$ & $319-1,115$ \\
\hline \multicolumn{5}{|l|}{ Go/NoGo ${ }^{b}$} \\
\hline Go & $99.17 \%(1.62)$ & $92.64-100$ & $390.77(45.88)$ & $325-518$ \\
\hline NoGo & $78.35 \%(12.66)$ & $41.27-95.52$ & $362.69(52.69)$ & $282-500$ \\
\hline IfGo & $99.31 \%(1.69)$ & $91.05-100$ & $441.79(58.87)$ & $343-558$ \\
\hline
\end{tabular}

Reaction times are presented in milliseconds. RTs, reaction times. ${ }^{\mathrm{a}} n=47 .{ }^{\mathrm{b}} n=48$. With regard to smoking cue-reactivity, no main effect of condition was observed for $\operatorname{RTs}\left(F[2,88]=1.01, p=.368, \eta^{2}=0.022\right)$. With regard to the Go/NoGo task, a main effect of condition was observed for accuracy $\left(F[1.02,44.82]=4.89, p=0.032 \eta^{2}=\right.$ $0.100)$ and $\operatorname{RTs}\left(F[1.63,73.40]=4.75, p=0.017, \eta^{2}=0.095\right)$. Accuracy rates for IfGo trials $(p<0.001)$ and Go trials $(p<0.001)$ were higher than for NoGo trials. RTs were slower on IfGo $(p<0.001)$ than NoGo and Go trials.

Table 4. Summary of linear regression analysis predicting NoGo accuracy

\begin{tabular}{|c|c|c|c|c|c|}
\hline Predictor & $\Delta R^{2}$ & $B$ & SE & $\beta$ & $p$ value \\
\hline \multicolumn{6}{|c|}{ Step 1 (control variables) } \\
\hline Constant & 0.081 & -0.023 & 1.792 & & 0.990 \\
\hline Pubertal status & & 5.458 & 4.739 & 0.167 & 0.256 \\
\hline Familial risk & & -0.899 & 0.650 & -0.201 & 0.173 \\
\hline \multicolumn{6}{|l|}{ Step 2} \\
\hline Constant & 0.025 & 0.007 & 1.787 & $7-$ & 0.997 \\
\hline Pubertal status & & 4.252 & 4.850 & 0.130 & 0.385 \\
\hline Familial risk & & -0.615 & 0.697 & -0.138 & 0.382 \\
\hline ETS exposure & & -0.326 & 0.294 & -0.177 & 0.273 \\
\hline
\end{tabular}

$n=48$. ETS, environmental tobacco smoke.

during task performance; for details of the complete network, see uploaded T-maps. Second, we examined whether there were significant associations between ETS exposure and brain activation associated with smoking cue-reactivity on the whole brain level as well as for the predefined ROIs using SVCs. These analyses did not reveal significant associations either at the whole brain level or within the ROIs. We further examined these null findings in the predefined ROIs by extracting the beta weights and applying Bayesian statistics. Bayes factors for ACC, striatum, VMPFC, amygdala, and insula were $\mathrm{BF}_{01}=12.22, \mathrm{BF}_{01}=4.64, \mathrm{BF}_{01}=4.14, \mathrm{BF}_{01}=3.57$, and $\mathrm{BF}_{01}=8.43$, respectively. Bayes factors indicated sub- stantial evidence for all null hypotheses, except the ACC, where strong evidence was observed for the null hypothesis.

Third, we exploratory examined the brain response for the smoke $>$ neutral contrast to test the brain response of nonsmoking adolescents toward smoking pictures regardless of ETS exposure. The results show more activity in the visual cortex, precuneus and fusiform gyrus, hippocampus, insula, posterior cingulate and frontal regions (superior frontal gyrus, inferior frontal gyrus, and medial frontal gyrus) for smoking pictures compared to neutral pictures.

\section{Inhibitory Control}

First, we examined brain responses to No_Gocorrect $>$ Go_correct to evaluate whether our task activated the expected brain regions. The results show activation within the frontal parietal cognitive control network including subcortical regions during task performance. Second, we examined whether there were significant associations between ETS exposure and brain activation related to inhibitory control on the whole brain level as well as for the predefined ROIs using SVCs. These analyses did not reveal significant associations either at the whole brain level or within the ROIs. Bayes factors for putamen, $\mathrm{ACC} /$ pre-SMA, IFG, and DLPFC were $\mathrm{BF}_{01}=$ $4.39, \mathrm{BF}_{01}=8.65, \mathrm{BF}_{01}=5.63$, and $\mathrm{BF}_{01}=7.64$, respectively, indicating substantial evidence for all null hypotheses. 


\section{Discussion}

This study investigated whether current ETS exposure is associated with brain functioning related to smoking cue-reactivity and inhibitory control in nonsmoking adolescents. Frequentist statistics did not show any evidence for associations between current ETS exposure and brain activation related to smoking cue-reactivity and inhibitory control. In addition, Bayesian statistics showed substantial-to-strong evidence for all null hypotheses. Therefore, we may conclude that given the current study design and study population, the associations between current ETS exposure and brain activation related to smoking cue-reactivity and inhibitory control are nonexisting.

\section{Smoking Cue-Reactivity}

The absence of an association between ETS and smoking cue-reactivity related brain activation suggests that repeated ETS exposure (i.e., in their lives right now) in nonsmokers is not associated with incentive salience of smoking cues. In general, repeated substance use causes substance-related cues to become conditioned cues. In turn, the motivational network becomes sensitized to substancerelated cues and causes increased activity in this system [27]. Our results show that there is no relation between these substance-related cues and current ETS exposure.

Substantial-to-strong evidence for the lack of an association between ETS and smoking cues might be due to the design of the cue-reactivity task and more specifically the fact that we used a standardized smoking picture set. Given that ETS exposure is often less frequent than smoking itself, it may be that incentive salience develops way slower and consequently generalization to standard smoking cues does not occur. Therefore, a standardized picture might not be as effective in evoking neural responses associated with ETS than more individually tailored pictures showing environments where individuals were exposed to nicotine inhalation. A study by Conklin and colleagues [50] indeed showed that individually tailored pictures of the places where participants smoked were more likely to prompt craving than pictures displaying personal nonsmoking situations and nonpersonal proximal smoking cues (e.g., ashtray, cigarette, and a pack of cigarettes) [50]. This study implies that individually tailored stimuli may be more salient thereby suggesting that the use of such stimuli might also increase the likelihood of finding an association between smoking cue-reactivity and ETS exposure.

Alternatively, substantial-to-strong evidence for the lack of an association between ETS exposure and smok- ing cue-reactivity may be due to the fact that the majority of our participants were female (70\%). Sex differences exist for the reinforcing and rewarding effects of nicotine. Research indicated that in comparison to women, men showed greater reward-related brain reactivity to smoking-related cues in brain regions [51]. Overall, the minority of men in this sample together with the lack of salience in nonsmokers in response to a standardized picture set of smoking-related cues might explain why we found substantial-to-strong evidence for the lack of an association between current ETS exposure and brain activation related to smoking cue-reactivity.

Although the results show an increased likelihood for no association between current ETS exposure and smoking cue-reactivity, neuronal cue-reactivity to smokingcues in all nonsmoking adolescents was observed in brain regions associated with attention, reward, and goal-directed behavior. More specifically, increased brain activity in the visual cortex, precuneus, and fusiform gyrus was observed, reflecting increased allocation of visual attention to smoking relative to neutral cues. Moreover, neuronal cuereactivity activity in the insula and hippocampus reflects enhanced reward/emotional processing [52]. Neuronal cue-reactivity to smoking cues in nonsmokers has been observed previously by Vollstadt-klein and colleagues [53]. Smokers perceive smoking cues as salient/pleasant, probably because of incentive salience through years of repeated associations with the delivery of nicotine, resulting in associated brain activity. Increased neuronal activity for smoking cues in similar brain regions, regardless of ETS exposure, for nonsmokers may suggest that smoking cues are perceived as salient in nonsmokers as well. However, 2 recent studies indicate that never-smokers might perceive cigarette cues as unpleasant $[54,55]$ so the observed brain activation for smoking cues may also be interpreted as reflecting the aversive nature of these stimuli. Future (longitudinal) research including valence and arousal ratings is needed to investigate participants' attitude toward smoking cues as well as related brain activity and its relation with ETS exposure in nonsmokers, as well as potential consequences for future smoking behavior.

\section{Inhibitory Control}

Substantial evidence was found for the absence of an association between current ETS exposure and inhibitory control for both the behavioral and neural level. This stands in contrast with previous research showing reduced inhibitory control in smokers as well as individuals prenatally exposed to cigarette smoke [11, 12, 22, 23]. Generally, it remains unclear whether deficits related to 
inhibitory control should be treated as a consequence of substance abuse, or whether these deficits are pre-existing and perhaps contributory to the development of addiction. Longitudinal and family studies found evidence that reduced inhibitory control might serve as a predetermined risk factor for addiction [56-58]. Specifically, Anokhin and Golosheykin [56] found that deficient frontal neuronal activity, related to response inhibition at age 14, was associated with increased risk for future nicotine dependence in adolescents at age 18. This suggests that these deficits are a cause of substance dependence rather than a consequence that may be (at least partially) genetically determined. If deficits in inhibitory control are mainly a predetermined risk factor for the development of substance use, which is partially genetically determined, rather than the consequence of nicotine intake, this might explain why we did not find an association between inhibitory control and current ETS exposure. To control for genetic confounding, we used the familial risk questionnaire, although future studies should use a genetically informative study design. It should be noted though that the mean familial risk score in the current study was rather low which might suggest that we did not include a group of participants with a very high genetic risk.

Another explanation could be the ongoing brain development during adolescence. Given the continued brain maturation in prefrontal regions, it may be that variability in developmental stage may have interfered with potential ETS effects in the current study. To capture this ongoing pubertal maturation, we, however, included PDS scores in our analyses [37].

\section{Strengths and Limitations}

To our knowledge, this study is the first to address the relation between current ETS exposure and brain activation related to cue-reactivity and inhibitory control using fMRI. The current study restricted analyses to participants who never had smoked a complete cigarette, thereby ruling out possible effects of early smoking on brain functioning. In addition, despite challenges with recruitment of this group, about half of the participants were in a lower educational track. Given that smoking rates are higher in the environment of individuals with lower educational levels $[59,60]$, these individuals are a risk group not only for the development of nicotine dependence but also for exposure to ETS.

Nevertheless, there are some limitations that warrant further discussion. The null findings in this study could be due to the observed levels of ETS exposure within the current sample of participants. ETS exposure in this group turned out to be low to moderate, with relatively few participants who had moderate-to-high exposure. While this homogeneous distribution may be in line with the decreasing levels of ETS exposure in the Dutch adolescent population, it may also explain the absence of the association between ETS exposure and brain functioning. A more heterogeneous sample including no, low, moderate, and especially high exposure to ETS should be incorporated in future studies to cover the whole range of ETS exposure. Furthermore, selecting a participant group with a smaller age range reducing the effect of developmental changes may increase the ability to observe associations with ETS.

Other limitations arise from the measurement of ETS exposure. The current study focused on current ETS exposure and therefore did not take lifetime ETS exposure into account. While standardized ETS exposure questionnaires are currently lacking, a similar method to ours was used in another study [61]. To further improve the ETS exposure measure, future research should include questions on the severity of exposure (i.e., time spent with various people in the environment as well as the average amount of cigarettes smoked when around) as well as lifetime ETS exposure (i.e., years of exposure) next to the frequency of ETS exposure. In order to obtain an even more accurate measure of actual exposure, future studies could include silicone wristbands measuring the nicotine exposure in the environment [62]. To measure lifetime ETS exposure and its associations with cue-reactivity and inhibitory control it would be most ideal to include ETS exposure measures in large longitudinal studies over the lifespan. This will result in a better measure of the accumulated exposure. For example, it would allow us to test whether repeated exposure over the years results in changes in smoking cue-reactivity and associated neural activity.

In conclusion, our results demonstrated that with this design, it is likely that current low-to-moderate ETS exposure is not associated with brain activation related to smoking cue-reactivity and inhibitory control. We recommend more research in large samples of adolescents with higher ETS exposure levels in longitudinal study designs before strong conclusions can be formulated.

\section{Statement of Ethics}

Written informed consent was obtained from all participants and from parents for participants younger than 16 years old. The study was approved by the ethical committee CMO ArnhemNijmegen (project \#2016-2409, 2015-2120). 


\section{Conflict of Interest Statement}

The authors have no conflicts of interest to declare.

\section{Funding Sources}

This research was funded by a VENI grant (No. 451-15-029) from the Netherlands Organisation for Scientific Research (NWO) to XXXXX. NWO had no further role in study design; in the collection, analysis, and interpretation of data; in the writing of the report; and in the decision to submit the paper for publication. This research was part of a larger project funded by a Dutch cancer society grant (No. Ti 2014-6984).

\section{Author Contributions}

M.L. wrote the grant for this project, designed the study, contributed to the data analysis, and edited the manuscript. J.D. designed the study, performed the test sessions and data analyses, and edited the manuscript. A.B. performed the test sessions and data analysis and wrote the manuscript. M.K. and R.O. wrote the grant for this project, designed the study, and edited the manuscript.

\section{References}

1 Öberg M, Jaakkola MS, Woodward A, Peruga A, Prüss-Ustün A. Worldwide burden of disease from exposure to second-hand smoke: a retrospective analysis of data from 192 countries. Lancet. 2011;377(9760):139-46.

2 Ter Weijde WR, Croes EA, Verdurmen JEE, Monshouwer K. Factsheet meeroken. Utrecht: Trimbos-Instituut; 2015.

3 Moritsugu KP. The 2006 report of the surgeon general: the health consequences of involuntary exposure to tobacco smoke. Am J Prev Med. 2007;32(6):542-3.

4 World Health Organization. International consultation on environmental tobacco smoke (ETS) and child health. Geneva: World Health Organization; 1999.

5 Bandiera FC, Richardson AK, Lee DJ, He JP, Merikangas KR. Secondhand smoke exposure and mental health among children and adolescents. Arch Pediatr Adolesc Med. 2011; 165(4):332-8

6 Moya J, Bearer CF, Etzel RA. Children's behavior and physiology and how it affects exposure to environmental contaminants. Pediatrics. 2004;113(4 Suppl 1):996-1006.

7 Willers S, Skarping G, Dalene M, Skerfving S. Urinary cotinine in children and adults during and after semiexperimental exposure to environmental tobacco smoke. Arch Environ Health. 1995;50(2):130-8.

8 Casey BJ, Getz S, Galvan A. The adolescent brain. Dev Rev. 2008;28(1):62-77.

9 Arain M, Haque M, Johal L, Mathur P, Nel W, Rais A, et al. Maturation of the adolescent brain. Neuropsychiatr Dis Treat. 2013;9:44961.

10 Rauh AV, Margolis A. Research review: environmental exposures, neurodevelopment and child mental health: new paradigms for the study of brain and behavioural effects. J Child Psychol Psychiatry. 2016;57(7):775-93.

11 Bennett DS, Mohamed FB, Carmody DP, Bendersky M, Patel S, Khorrami M, et al. Response inhibition among early adolescents prenatally exposed to tobacco: an fMRI study. Neurotoxicol Teratol. 2009;31(5):283-90.
12 Longo CA, Fried PA, Cameron I, Smith AM. The long-term effects of prenatal nicotine exposure on response inhibition: an fMRI study of young adults. Neurotoxicol Teratol. 2013; 39:9-18.

13 Bélanger M, O'Loughlin J, Okoli CT, McGrath JJ, Setia M, Guyon L, et al. Nicotine dependence symptoms among young never-smokers exposed to secondhand tobacco smoke. Addict Behav. 2008;33(12): 1557-63.

14 Okoli CT, Richardson CG, Ratner PA, Johnson JL. Non-smoking youths' "perceived" addiction to tobacco is associated with their susceptibility to future smoking. Addict Behav. 2009;34(12):1010-6.

15 Schuck K, Kleinjan M, Otten R, Engels RC, Difranza JR. Responses to environmental smoking in never-smoking children: can symptoms of nicotine addiction develop in response to environmental tobacco smoke exposure? J Psychopharmacol. 2013;27(6):53340.

16 Al-Delaimy W, Fraser T, Woodward A. Nicotine in hair of bar and restaurant workers. $\mathrm{N}$ Z Med J. 2001;114(1127):80-3.

17 Dimich-Ward H, Gee H, Brauer M, Leung V. Analysis of nicotine and cotinine in the hair of hospitality workers exposed to environmental tobacco smoke. J Occup Environ Med. 1997;39(10):946-8.

18 Brody AL, Mandelkern MA, London ED, Khan A, Kozman D, Costello MR, et al. Effect of secondhand smoke on occupancy of nicotinic acetylcholine receptors in brain. Arch Gen Psychiatry. 2011;68(9):953-60.

19 Chase HW, Eickhoff SB, Laird AR, Hogarth L. The neural basis of drug stimulus processing and craving: an activation likelihood estimation meta-analysis. Biol Psychiatry. 2011; 70(8):785-93.

20 Engelmann JM, Versace F, Robinson JD, Minnix JA, Lam CY, Cui Y, et al. Neural substrates of smoking cue reactivity: a meta-analysis of fMRI studies. Neuroimage. 2012;60(1): 252-62.
21 Kühn S, Gallinat J. Common biology of craving across legal and illegal drugs: a quantitative meta-analysis of cue-reactivity brain response. Eur J Neurosci. 2011;33(7):1318-26.

22 Luijten M, Machielsen MW, Veltman DJ, Hester R, de Haan L, Franken IH. Systematic review of ERP and fMRI studies investigating inhibitory control and error processing in people with substance dependence and behavioural addictions. J Psychiatry Neurosci. 2014;39(3):149-69.

23 Smith JL, Mattick RP, Jamadar SD, Iredale JM. Deficits in behavioural inhibition in substance abuse and addiction: a meta-analysis. Drug Alcohol Depend. 2014;145:1-33.

24 Field M, Cox W. Attentional bias in addictive behaviours: a review of its development, causes, and consequences. Drug Alcohol Depend. 2008;97(1-2):1-20.

25 Goldstein RZ, Volkow ND. Dysfunction of the prefrontal cortex in addiction: neuroimaging findings and clinical implications. Nat Rev Neurosci. 2011;12(11):652-69.

26 Franken IH. Drug craving and addiction: integrating psychological and neuropsychopharmacological approaches. Prog Neuropsychopharmacol Biol Psychiatry. 2003; 27(4):563-79.

27 Volkow ND, Wang GJ, Telang F, Fowler JS, Logan J, Childress AR, et al. Cocaine cues and dopamine in dorsal striatum: mechanism of craving in cocaine addiction. J Neurosci. 2006;26(24):6583-8.

28 Volkow ND, Fowler JS, Wang GJ. The addicted human brain viewed in the light of imaging studies: brain circuits and treatment strategies. Neuropharmacology. 2004; 47 Suppl 1(1):3-13.

29 Luijten M, Littel M, Franken IH. Deficits in inhibitory control in smokers during a go/ NoGo task: an investigation using event-related brain potentials. Plos One. 2011;6(4): e18898. 
30 Nestor L, McCabe E, Jones J, Clancy L, Garavan $H$. Differences in "bottom-up" and "topdown" neural activity in current and former cigarette smokers: evidence for neural substrates which may promote nicotine abstinence through increased cognitive control. Neuroimage. 2011;56:2258-75.

31 De Ruiter MB, Oosterlaan J, Veltman DJ, Van den Brink W, Goudriaan AE. Similar hyporesponsiveness of the dorsomedial prefrontal cortex in problem gamblers and heavy smokers during an inhibitory control task. Drug Alcohol Depend. 2012;121(1-2):81-9.

32 Galván A, Poldrack RA, Baker CM, McGlennen KM, London ED. Neural correlates of response inhibition and cigarette smoking in late adolescence. Neuropsychopharmacology. 2011;36(5):970-8.

33 Akkermans SE, Luijten M, van Rooij D, Franken IH, Buitelaar JK. Putamen functional connectivity during inhibitory control in smokers and non-smokers. Addict Biol. 2016; 23(1):359-68

34 Holz NE, Boecker R, Baumeister S, Hohm E, Zohsel K, Buchmann AF, et al. Effect of prenatal exposure to tobacco smoke on inhibitory control: neuroimaging results from a $25-$ year prospective study. JAMA Psychiatry. 2014;71(7):786-96.

35 Harakeh Z, Engels RC, Vries H, Scholte RH. Correspondence between proxy and self-reports on smoking in a full family study. Drug Alcohol Depend. 2006;84(1):40-7.

36 Racicot S, McGrath JJ, O’Loughlin J. An investigation of social and pharmacological exposure to secondhand tobacco smoke as possible predictors of perceived nicotine dependence, smoking susceptibility, and smoking expectancies among never-smoking youth. Nicotine Tob Res. 2011;13(10):926-33.

37 Petersen AC, Crockett L, Richards M, Boxer A. A self-report measure of pubertal status: reliability, validity, and initial norms. J Youth Adolesc. 1988;17(2):117-33.

38 Vink JM, Willemsen G, Boomsma DI. Heritability of smoking initiation and nicotine dependence. Behav Genet. 2005;35(4):397-406.

39 Heatherton TF, Kozlowski LT, Frecker RC, Fagerstrom KO. The Fagerstrom test for nicotine dependence: a revision of the Fagerstrom Tolerance Questionnaire. Br J Addict. 1991; 86:1119-27.

40 Vink JM, Willemsen G, Beem AL, Boomsma DI. The Fagerström test for nicotine dependence in a Dutch sample of daily smokers and ex-smokers. Addict Behav. 2005;30(3):5759.

41 Saunders JB, Aasland OG, Babor TF, de la Fuente JR, Grant M. Development of the alcohol use disorders identification test (AUDIT): WHO collaborative project on early detection of persons with harmful alcohol consumption-II. Addiction. 1993;88:791-804.

42 Poser BA, Versluis MJ, Hoogduin JM, Norris DG. BOLD contrast sensitivity enhancement and artifact reduction with multiecho EPI: parallel-acquired in homogeneity-desensitized fMRI. Magn Reson Med. 2006;55(6): 1227-35.

43 Pruim RH, Mennes M, Buitelaar JK, Beckmann CF. Evaluation of ICA-AROMA and alternative strategies for motion artifact removal in resting state fMRI. Neuroimage. 2015;112:278-87.

44 Pruim RH, Mennes M, van Rooij D, Llera A, Buitelaar JK, Beckmann CF. ICA-AROMA: a robust ICA-based strategy for removing motion artifacts from fMRI data. Neuroimage. 2015;112:267-77.

45 Eklund A, Nichols TE, Knutsson H. Cluster failure: why fMRI inferences for spatial extent have inflated false-positive rates. Proc Natl Acad Sci U S A. 2016;113(28):7900-5.

46 Flandin G, Friston KJ. Analysis of family-wise error rates in statistical parametric mapping using random field theory. Hum Brain Mapp. 2019 May;40(7):2052-4.

47 Tzourio-Mazoyer N, Landeau B, Papathanassiou D, Crivello F, Etard O, Delcroix N, et al. Automated anatomical labeling of activations in SPM using a macroscopic anatomical parcellation of the MNI MRI single-subject brain. Neuroimage. 2002;15(1):273-89.

48 Wagenmakers EJ, Love J, Marsman M, Jamil T, Ly A, Verhagen J, et al. Bayesian inference for psychology. Part ii: example applications with jasp. Psychon Bull Rev. 2018;25(1):5876.

49 Wagenmakers EJ, Wetzels R, Borsboom D, Van Der Maas HLJ. Why psychologists must change the way they analyze their data: the case of psi: comment on Bem (2011). J Pers Soc Psychol. 2011 Mar;100(3):426-32.

50 Conklin CA, Perkins KA, Robin N, McClernon FJ, Salkeld RP. Bringing the real world into the laboratory: personal smoking and nonsmoking environments. Drug Alcohol Depend. 2010;111(1-2):58-63.

51 Dumais KM, Franklin TR, Jagannathan K, Hager N, Gawrysiak M, Betts J, et al. Multi- site exploration of sex differences in brain reactivity to smoking cues: consensus across sites and methodologies. Drug Alcohol Depend. 2017;178:469-76.

52 Kobiella A, Smolka MN. Biological research on addiction: chapter 64 . Neuroimaging of nicotine and tobacco smoking in humans. Dresden, Germany: Academic Press; 2013. Vol. 2.

53 Vollstadt-Klein S, Kobiella A, Bühler M, Graf C, Fehr C, Mann K, et al. Severity of dependence modulates smokers' neuronal cue reactivity and cigarette craving elicited by tobacco advertisement. Addict Biol. 2019;16(1):166-75.

54 Deweese MM, Codispoti M, Robinson JD, Cinciripini PM, Versace F. Cigarette cues capture attention of smokers and neversmokers, but for different reasons. Drug Alcohol Depend. 2018;185:50-7.

55 Dieleman J, Kleinjan M, Otten R, van Schie HT, Heuvelmans V, Luijten M. Effects of environmental tobacco smoke exposure on brain functioning in never-smoking adolescents. Brain Behav. 2020;10(8):e01619.

56 Anokhin AP, Golosheykin S. Neural correlates of response inhibition in adolescents prospectively predict regular tobacco smoking. Dev Neuropsychol. 2016;41(1-2):22-37.

57 Jentsch JD, Pennington ZT. Reward, interrupted: inhibitory control and its relevance to addictions. Neuropharmacology. 2014;76 Pt B:479-86.

58 Mahmood OM, Goldenberg D, Thayer R, Migliorini R, Simmons AN, Tapert SF. Adolescents' fMRI activation to a response inhibition task predicts future substance use. Addict Behav. 2013;38(1):1435-41.

59 Eccles J. Influences of parents' education on their children's educational attainments: the role of parent and child perceptions. Lond Rev Educ. 2005;3(3):191-204.

60 Hiscock R, Bauld L, Amos A, Fidler J. Munafò $\mathrm{M}$. Socioeconomic status and smoking: a review. Ann Ny Acad Sci. 2012;1248(1):107-23.

61 Ball J, Sim D, Edwards R. Addressing ethnic disparities in adolescent smoking: is reducing exposure to smoking in the home a key? Nicotine Tob Res. 2018;21(4):430-8.

62 Quintana PJE, Hoh E, Dodder NG, Matt GE, Zakarian JM, Anderson KA, et al. Nicotine levels in silicone wristband samplers worn by children exposed to secondhand smoke and electronic cigarette vapor are highly correlated with child's urinary cotinine. J Expo Sci Environ Epidemiol. 2019;29(6):733-41. 